Epoche und Oskulation I 89 I Nov. $7.0 \mathrm{~m} . \mathrm{Z}$. Berlin

$$
\begin{aligned}
& L=346^{\circ} 49^{\prime} 37^{\prime \prime} 3^{\circ} \\
& \pi=754735.42\} \text { I } 890.0 \\
& \delta=16250 \quad 18.50 \\
& i=103242.5^{8} \\
& \varphi=41620.79 \\
& \mu=616.589821 \\
& \log a=0.5066734 \text {. }
\end{aligned}
$$

\begin{tabular}{|c|c|c|c|c|c|c|c|}
\hline 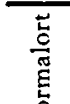 & $\begin{array}{c}\text { Datum } \\
2^{\text {h }} \mathrm{m} \cdot \mathrm{Z} \text {. Berlin }\end{array}$ & $\begin{array}{r}\text { Aus de } \\
\text { gungsgle }\end{array}$ & $\begin{array}{l}\text { Bedin- } \\
\text { ichungen }\end{array}$ & $\begin{array}{c}\text { I } \\
\text { Durch } \\
\text { Rech }\end{array}$ & $\begin{array}{l}\text { I. } \\
\text { direkte } \\
\text { nung }\end{array}$ & & $\begin{array}{l}\text { Se- } \\
\text { icht }\end{array}$ \\
\hline 2 & & $\Delta \alpha \cos 3$ & 18 & $\Delta \alpha \cos 8$ & $\Delta s$ & $p_{a}$ & $p d$ \\
\hline I & I 89 I Sept. 26 & +0.53 & -0.03 & +0.54 & -0.03 & 4 & 4 \\
\hline II & Okt. 5 & --2.91 & -3.80 & -2.79 & -3.77 & I & I \\
\hline III & 0 & -3.02 & +2.12 & -2.85 & +2.15 & I & I \\
\hline IV & 21 & +2.16 & +1.35 & +2.26 & + I.3I & I & $\mathbf{I}$ \\
\hline V & Nov. 5 & +1.90 & +1.35 & +1.93 & $+1 \cdot 36$ & I & I \\
\hline VI & 27 & +0.93 & -0.23 & +0.87 & -0.24 & I & $\mathbf{I}$ \\
\hline VII & Dez. I & +1.64 & +0.01 & +1.64 & -0.01 & I & $\mathbf{I}$ \\
\hline VIII & 1892 Nov. 2 & -1.20 & -0.22 & $-I \cdot 30$ & -0.29 & 2 & 2 \\
\hline IX & 1897 Sept. 2 & - & +0.31 & -4.94 & $+0.3^{2}$ & & 2 \\
\hline $\mathrm{X}$ & $3 c$ & $--x .86$ & -3.16 & -1.94 & --3.19 & & I \\
\hline XI & I 898 lez. I & +0.27 & -0.28 & +0.39 & -0.26 & I & I \\
\hline XII & 1903 Sept. 23 & : +0.13 & +0.79 & -0.04 & $1+0.77$ & $i 3$ & 3 \\
\hline
\end{tabular}

Diese Elemente lassen in den Beobachtungen mit Berücksichtigung der Störungen durch Jupiter und Saturn folgende Fehler im Sinne B-R übrig:

Bodenbach, 19 1 2 Juni 7 .
Aus den übrigbleibenden Fehlern I findet sich die Summe der Fehlerquadrate:

$$
[v v p]=7 \mathrm{r} .87
$$

und der mittlere Fehler einer Beobachtung vom Gewicht I :

$$
\varepsilon= \pm 2.056 \text {. }
$$

Die in $L, \pi$ und $\mu$ verbesserten Störungswerte für die Zeit I 89 I Nov. 7.0 bis 19 I 2 April I I.o lauten:

$$
\begin{array}{ll}
A L=+{ }^{\circ} 39^{\prime} 22^{\prime \prime 11} & A i=+0^{\circ} \circ^{\prime} 43^{\prime \prime} .98 \\
A \pi=+21939.28 & A \varphi=-05315.93 \\
A \delta=-020 \text { 1 } 2.95 & A \mu=+1.075892 .
\end{array}
$$

Die Präzession von 1890.0 bis 1910.0 beträgt:

$$
\begin{aligned}
& \Delta L=A \pi=+16^{\prime} 45^{\prime \prime 2} 29 \\
& \Delta \delta=+1635.49 \\
& A i=-09.25
\end{aligned}
$$

Für die Epoche und Oskulation I9 I 2 April Ir.o m. Z. Berlin ergeben sich demnach die folgenden Elemente:

$$
\left.\begin{array}{rl}
L & =186^{\circ} 28^{\prime} 24^{\prime \prime} .76 \\
\pi & =782359.99 \\
\delta & =1624641.04 \\
i & =103317.31
\end{array}\right\} \text { 19 } 10.0
$$

Diese neuen Elemente lassen in den Beobachtungen Wien 1912 April 6 und 10 nachstehende Fehler im Sinne $\mathrm{B}-\mathrm{R}$ übrig:

$$
\begin{aligned}
1912 \text { April } 6+0.61 & -3.9 \\
\text { 10 }+0.45 & -1.5 \\
& \text { H. Mader. }
\end{aligned}
$$

\title{
Identité certaine de la comète 1912b (Schaumasse) et de la comète Tuttle.
}

Par M. M. Fayet et Schaumasse.

Nous avons utilisé dans ce nouveau calcul, les observations suivantes, obtenues à l'observatoire de Nice par $M$. Schaumasse:

\begin{tabular}{|c|c|c|c|c|c|c|c|c|c|c|c|}
\hline IC & & $d \alpha$ & $\Delta \delta$ & 1. & $\boldsymbol{\alpha}$ app. & $|\log p \cdot \Delta|$ & & & $|\log p \cdot \Delta|$ & . & \\
\hline & $\begin{array}{l:lll}7 & 6^{\mathrm{h}} & 57^{\mathrm{m}} & 3^{\mathrm{s}} \\
9 & 6 & 59 & 30\end{array}$ & $\begin{array}{l}+I^{m} 26.26 \\
-I 57.36\end{array}$ & $\begin{array}{l}+5^{\prime} \text { I } 5^{\prime \prime} 6 \\
-28 \\
2.9\end{array}$ & $\begin{array}{l}2 s, 2 p \\
4 s, 4 p\end{array}$ & 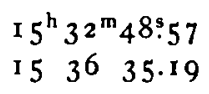 & - & + & $\begin{array}{lll}1^{\circ} & 36^{\prime} & \text { I } \\
& 5 \cdot 7 \\
3 & 54 & 37 \cdot 3\end{array}$ & $\begin{array}{l}- \\
--\end{array}$ & $\begin{array}{l}+1.08 \\
+1.07\end{array}$ & \\
\hline
\end{tabular}

I 912 t. m. Paris

Oct. 18.69092 23.65749 28.67769

Nov. 1.67147

$$
\begin{aligned}
& \text { I.ongitude géoc. 1912.0 Latitude géoc. } 1912.0 \\
& \text { I } 50^{\circ} 56^{\prime} 51^{\prime \prime} .8 \\
& \text { I } 56 \quad 2744.9 \\
& \text { - } 10^{\circ} \text { I I' } 37^{\prime \prime} .6 \\
& -143231.5 \\
& \begin{array}{lll}
-18 & 52 & 55 \cdot 7
\end{array} \\
& \begin{array}{lll}
-22 & 12 & 52.2
\end{array}
\end{aligned}
$$

En employant la méthode de la variation des distances géocentriques et en partant des observations des 18 et 28 oct., nous avons obtenu le système suivant:

$$
\left.\begin{array}{rl}
T & =1912 \text { Oct. } 28.41055 \text { t. m. Paris } \\
\delta & =269^{\circ} 33^{\prime} 57^{\prime \prime} .0 \\
\pi & =1162526.5 \\
i & =55023.8
\end{array}\right\} \text { I912.0 }
$$

Représentation des lieux moyens:

$$
\mathrm{O}-\mathrm{C}\left\{\begin{array}{rrr}
\cos \beta \mathrm{d} \lambda & +0.3 & \text { Nov. I } \\
\mathrm{d} \beta & -1.3 & -\mathrm{o.4} 4 \\
& -1.5
\end{array}\right.
$$

Voici enfin la comparaison des éléments précédents avec ceux que l'on obtient pour la comète Tuttle, si l'on tient compte des perturbations approchées, que j'ai calculées, concernant l'action de Jupiter pendant la période I900-I 90 I comète 1912 b comète Tuttle

$$
\begin{aligned}
& \delta=269^{\circ} 34^{\prime \circ} \\
& \pi=\text { I } 625.4 \\
& i=\begin{array}{ll}
55 & 0.4
\end{array} \\
& e=0.8055^{2}
\end{aligned}
$$

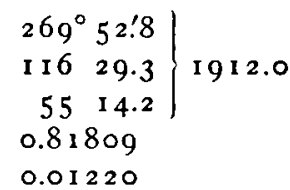

L'identité n'est donc plus douteuse.

Observatoire de Nice, Ig I 2 Nov. 3.

G. Fayet.

Komet 1912a (Gale).

Beobachtungen am Heliometer der Sternwarte Bamberg von Prof. E. Hartuig, 